\title{
Development and Validation of LC-MS/MS Method for Determination of Trimipramine Maleate in Human Plasma
}

Venkata Sairam Koganti*, Sekuri Shashidhar Reddy, Jyothigoudapura Channabasappa Thejaswini and Gurupadayya Bannimath

Department of Pharmaceutical Analysis, JSS College of Pharmacy, JSS University, Mysore 570 015, India

\begin{abstract}
A simple, sensitive, Liquid Chromatography-Tandem Mass Spectrometry (LC-MS/MS) method was developed for the determination of Trimipramine maleate in human plasma using Opipramol dihydrochloride as internal standard (ISTD). The analyte and ISTD were isolated from plasma by protein precipitation method using methanol. The chromatographic separation was carried out on ZORBAX ECLIPSE XDB- C18 column (4.6 x $150 \mathrm{~mm}, 5 \mu \mathrm{m})$. The elution was done with isocratic mobile phase composed of $5 \mathrm{mM}$ ammonium formate with $0.1 \%$ formic acid and methanol in the ratio of $25: 75(\mathrm{v} / \mathrm{v})$ at a flow rate of $1.0 \mathrm{ml} / \mathrm{min}$. Trimipramine and Opipramol were detected with proton adducts at $\mathrm{m} / \mathrm{z} 295.20 \rightarrow 100.10$ and $\mathrm{m} / \mathrm{z} 364.30 \rightarrow 171.20$ in multiple reaction monitoring (MRM) positive mode respectively. The retention time of Trimipramine and ISTD were found to be $1.67 \mathrm{~min}$ and $1.48 \mathrm{~min}$ respectively. The calibration curves were linear over concentration range of 0.1 to $100.1 \mathrm{ng} / \mathrm{ml}$. The lower limit of quantification was found to be $0.1 \mathrm{ng} / \mathrm{ml}$. The method was validated by EMEA (European medicines agency) guidelines for linearity, accuracy and precision, matrix factor, recovery, selectivity and stability. The validation parameters were found to be well within the acceptance limit.
\end{abstract}

Keywords: LC-MS/MS; Human plasma; Trimipramine maleate; Multiple reaction monitoring; Opipramoldihydrochloride

Abbreviations: \%: Percent; Cmax: Concentration Maximum; EMEA: European Medicines Agency; LC-MS/MS: Liquid Chromatography-Mass Spectrometry/Mass Spectrometry; LLOQ: Lower Limit of Quantitation; LMQC: Lower Medium Quality Control; LOD: Limit of Detection; LOQ: Limit of Quantification; LOQ QC: Limit of Quantification Quality Control; LQC: Low Quality Control; MQC: Medium Quality Control; MRM: Multiple Reaction Monitoring; Psi: Pounds per square inch; Q1: First Quadrupole; Q3:Third Quadrupole; rpm: Rotations per minute; V: Voltage

\section{Introduction}

Trimipramine maleate is chemically dihydrobenzo [b] [1] benzazepin-11-yl)-N,N,2-trimethylpropan-1-amine (Figure 1) used as a tricyclic antidepressant (TCA) [1]. These agents act by blocking the reuptake of serotonin and norepinephrine; the brain chemicals that are related to mood. By blocking the reabsorption of these chemicals, cyclic antidepressants increase the levels of serotonin and norepinephrine which helps in elevating the mood.

Trimipramine (marketed forms Surmontil, Rhotrimine, Stangyl) is a tricyclic antidepressant (TCA) with sedative properties. Trimipramine is primarily used in the treatment of major depressive disorder [2] especially where sedation is required due to its prominent sedative effects [3]. Generally, depression disorders are associated with an increase in the risk of suicide [4] and monitoring trimipramine plasma level concentration would be helpful in optimizing the dose for enhancement of clinical usage [5]. Quantifying the plasma drug would be an add-on for therapeutic drug monitoring and useful in pharmacokinetic studies in treatment of depression.

Several spectrometric [6,7], chromatographic techniques have been reported, including UPLC methods [8] but till date there were no perfect LC-MS/MS reported methods. But all these methods are inadequate in terms of sensitivity and employed uneconomical extraction procedures. The current method employed extraction procedure using methanol yielding good recovery results. Method development was done using opipramol dihydrochloride (Figure 2) as an internal standard, unlike deuterated standards and obtaining aqueous linearity regression $\mathrm{r}=0.9993$.

\section{Experimental}

\section{Chemicals and reagents}

Trimipramine maleate and Opipramoldihydrochloride were obtained from RL Fine Chemicals Ltd., Bangalore. Methanol of analytical gradient was purchased from Sigma Aldrich, Ammonium Formate was purchased from Fischer Scientific and Milli-Q water was used. Blank human plasma was obtained from volunteers of human pathological unit.

\section{Instrumentation}

The LC-MS/MS system consisted of a High Performance Liquid Chromatography (Schimadzu LC-20AD, autosampler SIL-30ACMP) coupled with a Sciex Triple Quadrupole Mass Spectrometer (API 4000, MDS, Sciex, Ontario-Canada) equipped with an electrospray ionization (ESI) (Applied Biosystems). Data acquisition and processing were controlled by Applied Biosystems/MSD SCIEX Analyst software (Version 1.6.1).

\section{Chromatographic conditions}

Chromatographic separations were achieved by ZORBAX Eclipse XDB-C18 (4.6 X $100 \mathrm{~mm} ; 3.5 \mu)$ column with a flow rate of $1 \mathrm{ml} / \mathrm{min}$. Mobile phase was methanol: $5 \mathrm{mM}$ ammonium formate with $0.1 \%$

*Corresponding author: Venkata Sairam Koganti, Department of Pharmaceutical Analysis, JSS College of Pharmacy, JSS University, Shivarathreeshwara Nagar Mysore-570 015, India, Tel: 91-9739780422; E-mail: rx.sairam@gmail.com

Received July 16, 2015; Accepted August 21, 2015; Published August 24, 2015

Citation: Koganti VS, Reddy SS, Thejaswini JC, Bannimath G (2015) Development and Validation of LC-MS/MS Method for Determination of Trimipramine Maleate in Human Plasma. Pharm Anal Acta 6: 413. doi:10.4172/21532435.1000413

Copyright: @ 2015 Koganti VS, et al. This is an open-access article distributed under the terms of the Creative Commons Attribution License, which permits unrestricted use, distribution, and reproduction in any medium, provided the original author and source are credited. 
<smiles>CC(CN(C)C)CN1c2ccccc2CCc2ccccc21</smiles>

Figure 1 : Structure of Trimipramine.<smiles>C(=CN1c2ccccc2CCc2ccccc21)CN1CCNCC1</smiles>

Figure 2 : Structure of Opipramole.

formic acid in a ratio of 75:25. Injection volume was $10 \mu$ l. The run time was $3 \mathrm{~min}$.

\section{Mass spectrometry conditions}

Mass parameters were set and tuned in positive ion mode for Q1 and Q3 masses. The mass parameters were listed in Table 1. The full scan and product ion spectra of trimipramine are shown in (Figure 3).

\section{Preparation of stock and working standard solutions}

Stock of both trimipramine and internal standard were prepared by weighing $5 \mathrm{mg}$ of each and dissolving in 100\% methanol separately giving $5 \mathrm{mg} / \mathrm{ml}$ concentrations. Subsequently analyte stock concentration is further diluted by $60 \%$ methanol to get $756 \mu \mathrm{g} / \mathrm{ml}$ and internal standard to $200 \mathrm{ng} / \mathrm{ml}$.

\section{Preparation of calibration standards and quality control samples}

Calibration standards and quality control solutions were prepared based on the $\mathrm{C}_{\max }$ of the analyte [9]. Initially, all the stock solution was prepared in aqueous media. Now spiking of these aqueous stock solution into blank plasma were done to get the desired calibration standards of standards 8 to standard 1 of range 100.573, 80.458, 40.229, $20.115,8.046,3.218,0.209,0.105 \mathrm{ng} / \mathrm{ml}$ respectively. The quality control samples of HQC, MQC, LQC and LLOQC are of concentrations 75.619, $37.809,0.284,0.109 \mathrm{ng} / \mathrm{ml}$.

\section{Sample preparation}

Each plasma aliquot of $100 \mu \mathrm{l}$ was taken and to it $50 \mu \mathrm{l}$ of Internal standard drug was added and vortexed $(30 \mathrm{sec})$. Subsequently to it 400 $\mu \mathrm{l}$ of methanol was added and vortexed $(30 \mathrm{sec})$. Now these are cool centrifuged at $4500 \mathrm{rpm}$ for $5 \mathrm{~min}$. The supernatant is collected and 150 $\mu \mathrm{l}$ is transferred to autosamplers and $10 \mu \mathrm{l}$ is injected.

\section{Method Validation 5.1System Suitability}

To equilibrate chromatographic system, six replicate injections of reference standard solutions (MQC level concentration) have been injected.

\section{ASCOT (Auto sampler carry over test)}

To check the residual compound in standard blank from the previous injection, sequential injections of processed samples were given in the order of Diluent (mobile phase) $\rightarrow$ Standard blank $\rightarrow$ Standard $1 \rightarrow$ Standard $8 \rightarrow$ Standard blank (re-injected).

\section{Selectivity}

To evaluate any interference at retention time of analyte and ISTD, six different individual blank plasma lots (including 1 heamolysed and 1 lipemic) were screened. Six blanks and respective lower limit of quantitation (LLOQ) samples were processed and analyzed.

\section{Linearity}

Calibration curve covering the entire range of Trimipramine from $0.1 \mathrm{ng} / \mathrm{ml}$ to $100 \mathrm{ng} / \mathrm{ml}$ was analyzed each with eight concentration levels. Calibration curves covering the entire range of the analytical method were constructed with analyte area on $\mathrm{Y}$-axis and analyte concentration on $\mathrm{X}$-axis with the regression model (linear $1 / \mathrm{x}^{2}$ weighting $=\mathrm{mx}+\mathrm{c}$ ). The correlation coefficient was found to be 0.999 .

\section{Matrix effect}

It was investigated for six different individual plasma lots (4 normal, 1 haemolysed and 1 lipemic) at LQC and HQC concentration level. Six unextracted (aqueous) and six post extracted LQC and HQC samples were processed and analyzed. The matrix factor for analyte and ISTD should be within $\pm 15 \%$ and IS normalized matrix factor should be $\leq 15 \%$ at each level.

\section{Precision and accuracy}

Three different batches of precision and accuracy were performed, intra-assay precision was performed at LLOQ $(0.109 \mathrm{ng} / \mathrm{ml})$, LQC (0.284 ng/ml), MQC (37.809 ng/ml) and HQC (75.619 ng/ml) concentration levels for a six replicates in each analytical run. Interassay precision and accuracy were calculated after the replicates in three different analytical runs.

\section{Recovery}

The extraction efficiency of the analytical procedure was evaluated. By processing and analyzing six each LQC, MQC and HQC concentration level. By comparing the responses of analyte and ISTD in unextracted and extracted samples of equivalent concentration levels.

\begin{tabular}{|l|l|}
\hline Parameter & Value \\
\hline Source Temperature $\left.\mathbf{(}^{\circ} \mathbf{C}\right)$ & 500 \\
\hline Ion Source Gas1 $($ Psi) & 40.00 \\
\hline Ion Source Gas2 (Psi) & 45.00 \\
\hline Curtain gas (Psi) & 30.00 \\
\hline Collision gas (Psi) & 6.00 \\
\hline lon Spray Voltage (V) & 5500.00 \\
\hline Dwell time (ms) & 200.00 \\
\hline Entrance potential (V) & 10 \\
\hline Collision cell exit potential (V) & 10 \\
\hline MRM Transition (amu)-Trimipramine & $295.30 \rightarrow 100.10$ \\
\hline MRM Transition (amu)- Opipramol & $364.30 \rightarrow 171.20$ \\
\hline Collision Energy (V) & $25($ analyte)/ 30(IS) \\
\hline Declustering potential (V) & $50($ analyte)/ $80($ IS) \\
\hline
\end{tabular}

Table 1: Mass Spectrometer Parameters. 


\section{Stability studies}

Experiments were performed to evaluate the stability of analyte in plasma at different simulating conditions of processing. Freeze thaw, bench top and processed sample stability like in-injector and wet extract were performed at LQC and HQC levels in six replicates.

\section{Results and Discussion}

\section{Method development and optimization}

The chromatographic conditions were optimized with several trials while change in mobile phase composition to achieve symmetric peak shapes and good resolution. Mobile phase buffer composition was (ammonium formate and formic acid). Initially mobile phase methanol $0.1 \%$ formic acid $(80: 20)$ is used and the retention time was found to be $1.44 \mathrm{~min}$ for analyte and $1.52 \mathrm{~min}$ for ISTD, the detector response was found to be less and peak shape was not good. The mobile phase composition was altered in the next trial to Methanol:5 mm ammonium formate with $0.1 \%$ formic Acid (80:20) and retention time was found to be $1.38 \mathrm{~min}$ for analyte and $1.27 \mathrm{~min}$ for ISTD, the peak wasn't symmetric and to this mobile phase composition further modified by changingvolume proportion (65:35) which resulted in increase of retention time of analyte to $2.66 \mathrm{~min}$ and for ISTD $2.14 \mathrm{~min}$. Furthermore the composition proportion was changed to (75:25) methanol: buffer and retention time was found to be $1.67 \mathrm{~min}$ for analyte and $1.48 \mathrm{~min}$ for ISTD. This method was optimized as chromatographic conditions.

The mass parameters were tuned and detection was carried out in positive polarity with multiple monitoring reaction (MRM) modes. The mass parameters were tuned and optimized for Q1 (parent ion) and Q3 (daughter ion).

\section{Method validation}

The developed method was validated in terms of system suitability, auto sampler carry over test, selectivity, linearity, matrix effect, precision and accuracy, recovery and for stability.

System suitability: System suitability was performed by six replicate injections of aqueous MQC level concentration (AQS MIX). The acceptance criteria were met. The $\% \mathrm{CV}$ of retention time limit was found to be 0 and 0 for both analyte and ISTD and $\%$ CV was found to be 2.19 for response ratio limit. Which satisfactorily passed the criteria of $\% \mathrm{CV}$ for retention time and response ratio of analyte and ISTD NMT $5 \%$.

(ASCOT) Autosampler carryover test: Response of interference at retention times of Analyte and ISTD in re-injected blank sample after injecting extracted Standard- 1 and Standard- 8 was within the limits i.e. response of interference at analyte retention time NMT 20\% of LLOQ $(0.109 \mathrm{ng} / \mathrm{ml})$ response and NMT $5 \%$ for ISTD.

Selectivity: Selectivity was performed with extracted LLOQ samples and blank from each matrix were analyzed to evaluate selectivity. The $\%$ interference at the retention time of ISTD for all six biological matrices was found to be less than $20 \%$ of the mean LLOQ response $(0.109 \mathrm{ng} /$ $\mathrm{ml}$ ). The chromatograms of selectivity of Standard blank (Figure 4) of plasma lot1 and LLOQ (Figure 5) of plasma lot 1 are shown in.

Linearity: Calibration curve covering the entire range of Trimipramine from $0.1 \mathrm{ng} / \mathrm{ml}$ to $100 \mathrm{ng} / \mathrm{ml}$ was analyzed each with eight concentration levels. Calibration curves covering the entire range of the analytical method were constructed with analyte area on Y-axis and analyte concentration on $\mathrm{X}$-axis with the regression model (linear $1 / \mathrm{x}^{2}$ weighting $\left.=\mathrm{mx}+\mathrm{c}\right)$. The correlation coefficient was found to be 0.999. Calibration curve plot (Figure 6) and the chromatograms of standard-1 (Figure 7 and 8 ) and standard-8 are shown below.

Matrix effect: Processed and analyzed calibration standards in the same matrix which is to be used during validation experiment and also six different lots of plasma at LQC and HQClevels as per the procedure are described in the sample preparation section. \% Nominal concentration was found to be $99.3 \%$ (LQC) and $89.8 \%$ (HQC) for the dilutions, which passed the limit of $85-115 \%$.

Precision and accuracy: Three batches were performed using six replicates at concentration levels LLOQ, LQC, MQC, HQC levels. The within batch \% CV was $0.7 \%$ to $7.1 \%$ and $\%$ accuracy was found to be $88 \%$ to $108 \%$. The results were found to be within $\pm 15 \%$. The results of Intra batch precision and accuracy are tabulated in Table 2 and Inter batch precision accuracy is Table 3 .

Recovery: The percentage mean recovery for trimipramine was found to be $93.2 \%$. The mean recovery of the ISTD was found to be $100.4 \%$ at a concentration of $200 \mathrm{ng} / \mathrm{ml}$.

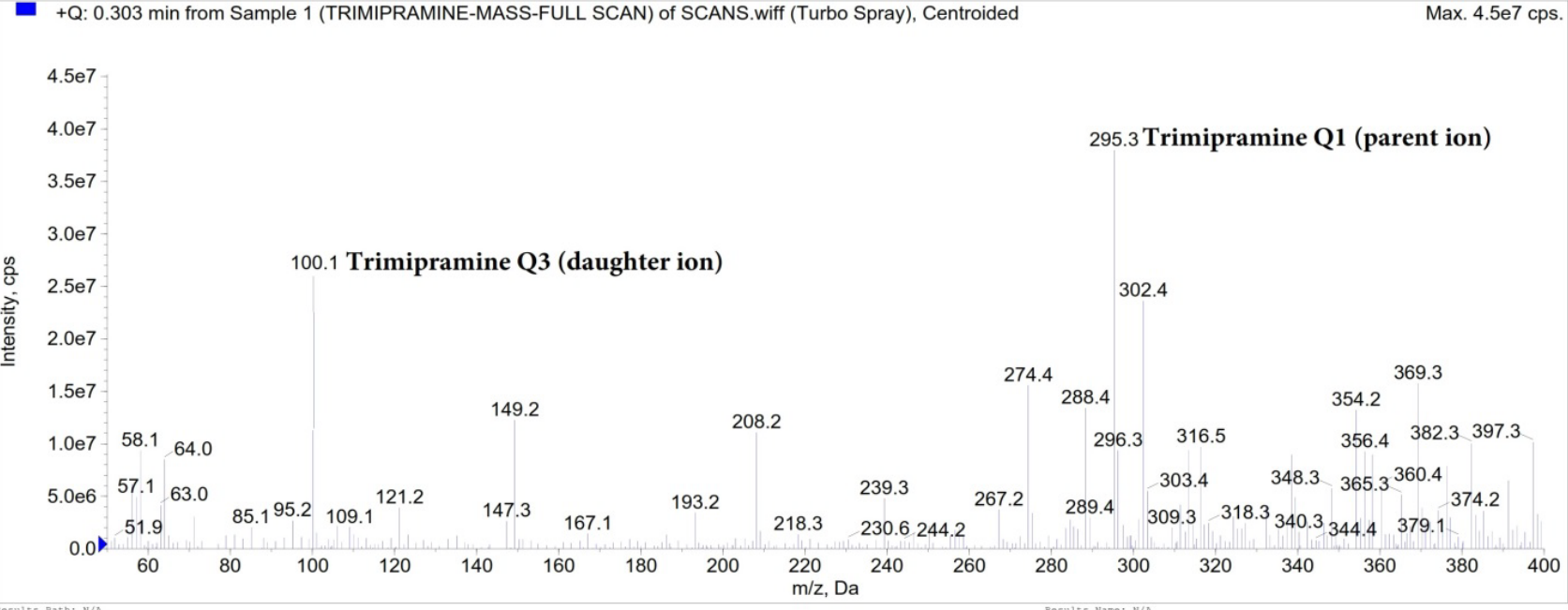

Figure 3: Full scan and product ion spectra of Trimipramine. 


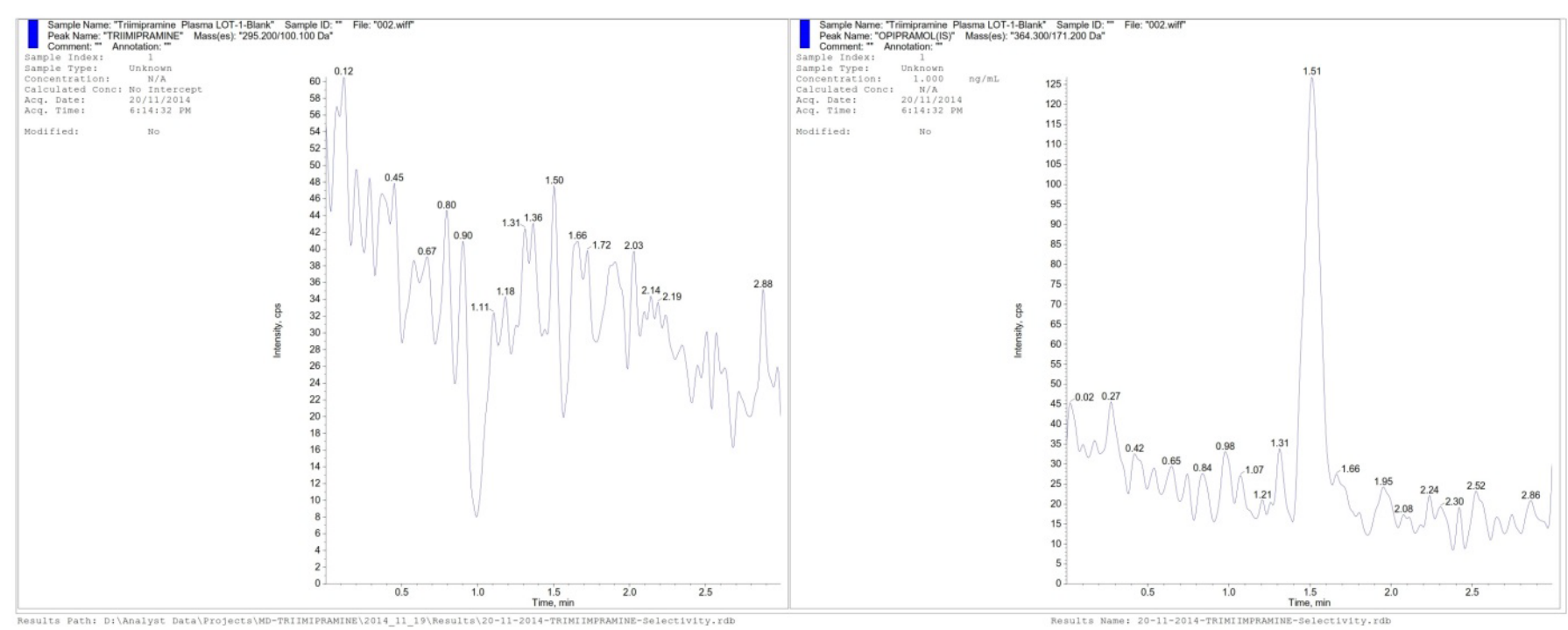

Figure 4: Selectivity- Plasma Lot 1-Standard Blank.

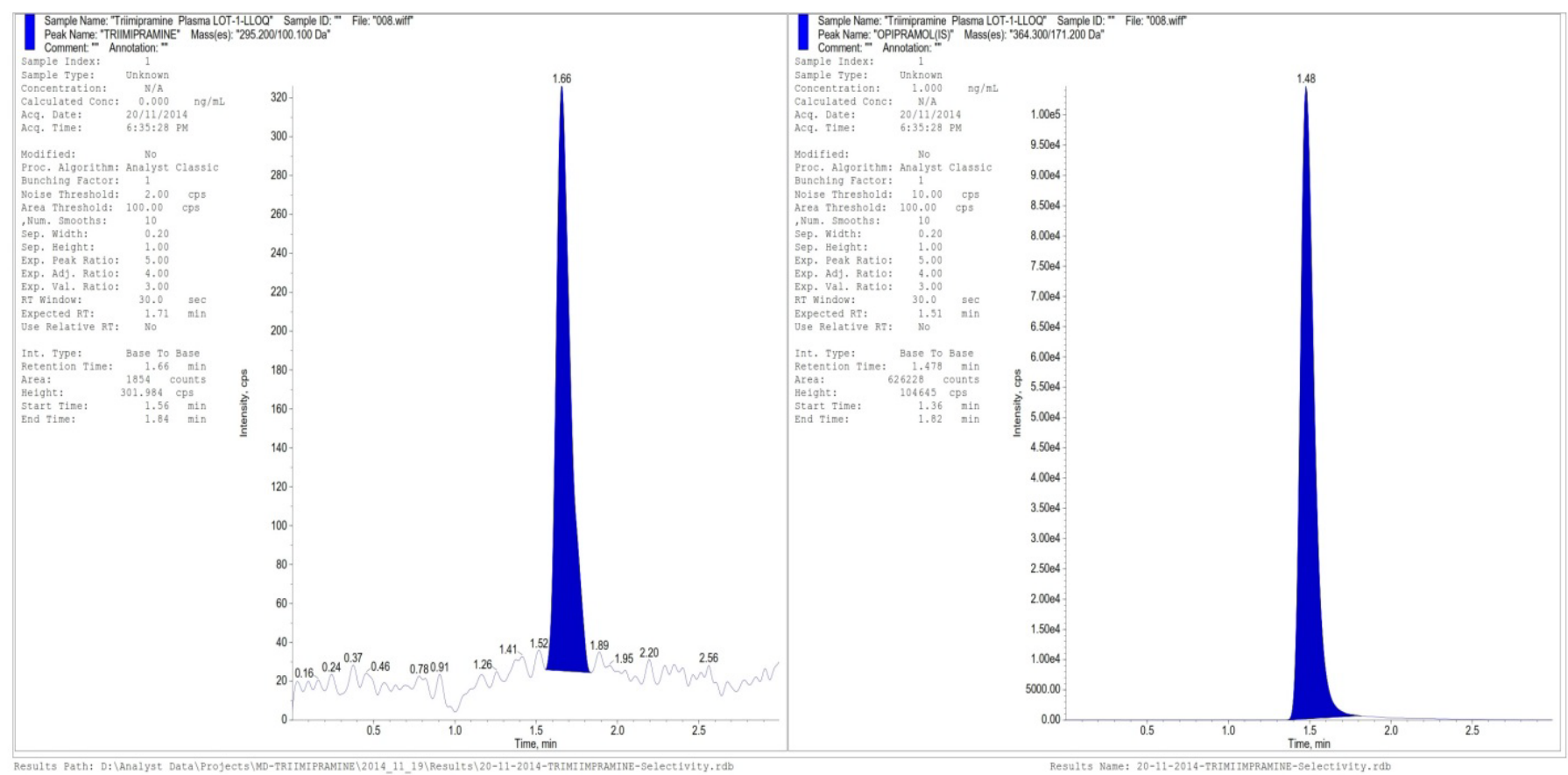

Figure 5: Selectivity-Plasma Lot 1- LLOQ.

Stability: Stability of analyte was assessed at all processing and sample storing conditions. The stability results are tabulated in Table 4.

Freeze thaw: The stability of analyte was determined in plasma during freeze-thaw cycles by freezing at $-70^{\circ} \mathrm{C}$ in freezer and thawing at room temperature for three cycles (interval of 12 hours) and thawing for minimum of one hour for every cycle. The precision was found to be $2.86 \%$ (LQC) and $4.27 \%$ (HQC) and mean \% bias was found to be -3.47 (LQC) and -5.03 (HQC). The results were found to be within $\pm 15 \%$.

Benchtop: The samples were stored unprocessed for 6 hours on bench at room temperature. The precision was found to be $3.07 \%$ (LQC) and 3.5\% (HQC) and mean \% bias was found to be 3.3\% (LQC) and $-2.02 \%$ (HQC).
In-injector: The processed sample was stored at autosampler temperature $\left(4^{\circ} \mathrm{C}\right)$ for a period of 12 hours. The precision was found to be $1.8 \%$ (LQC) and $0.9 \%$ (HQC) and mean \% bias was found to be $-4.7 \%$ (LQC) and $-5.5 \%$ (HQC).

Wet extract: The processed sample was stored at room temperature for a period of 12 hours. The precision was found to be $1.9 \%$ (LQC) and $1.2 \%$ (HQC) and mean \% bias was found to be $9.3 \%$ (LQC) and $8.1 \%$ (HQC).

\section{Conclusion}

A sensitive, specific, accurate and reproducible LC-MS/MS method has been developed employing a simple protein precipitation 


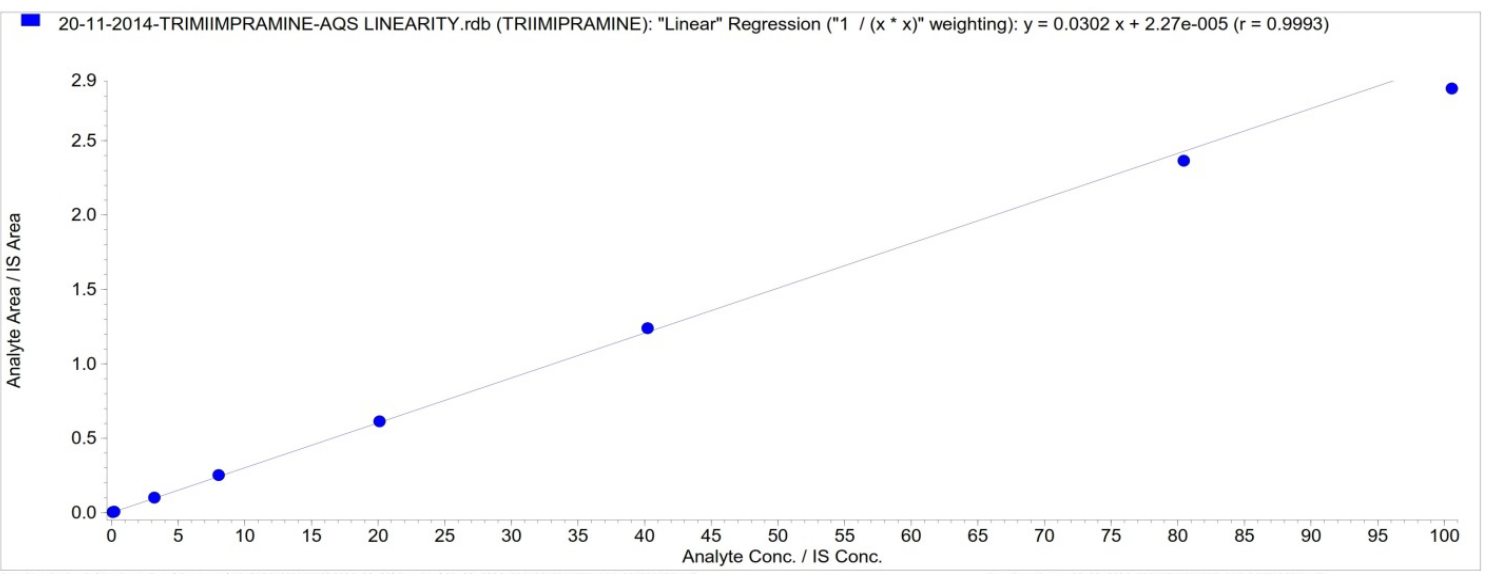

Figure 6: Linearity Calibration Curve.
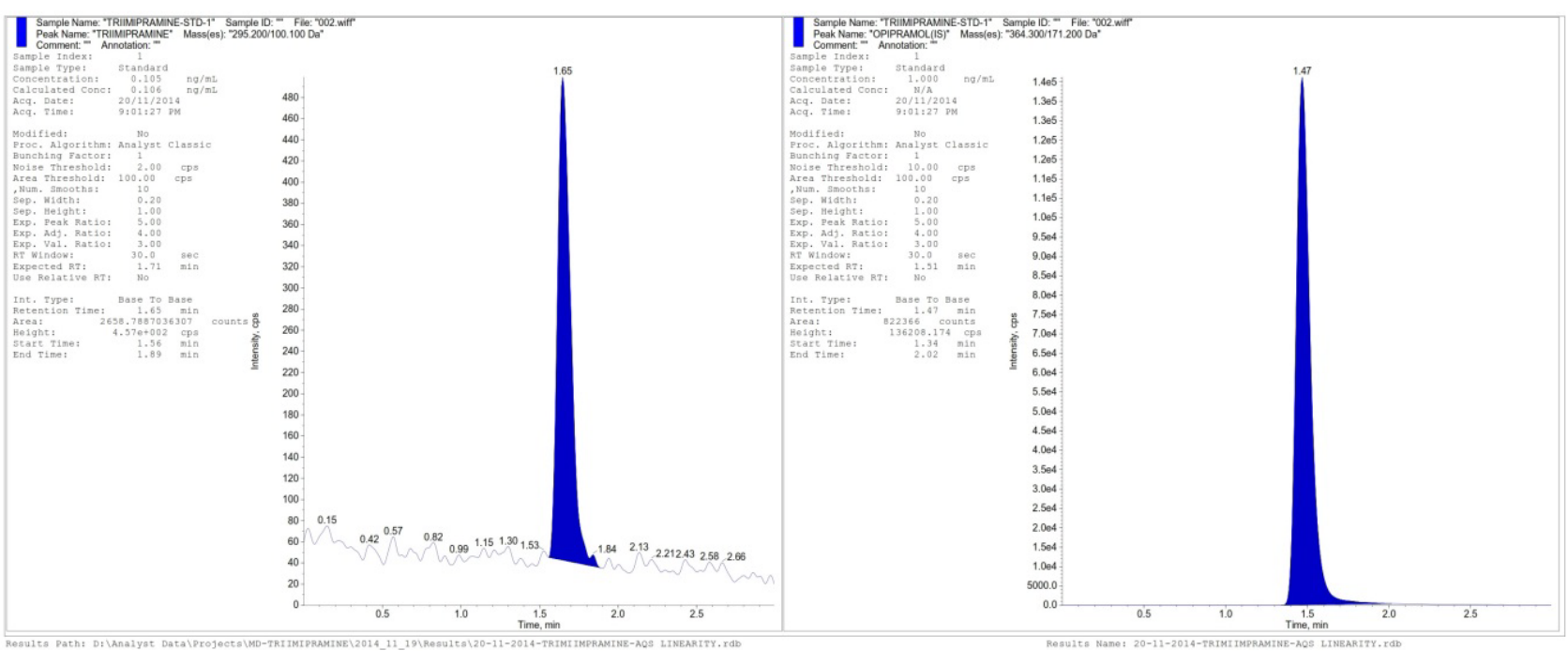

Figure 7: Linearity-Standard-1 Chromatogram.
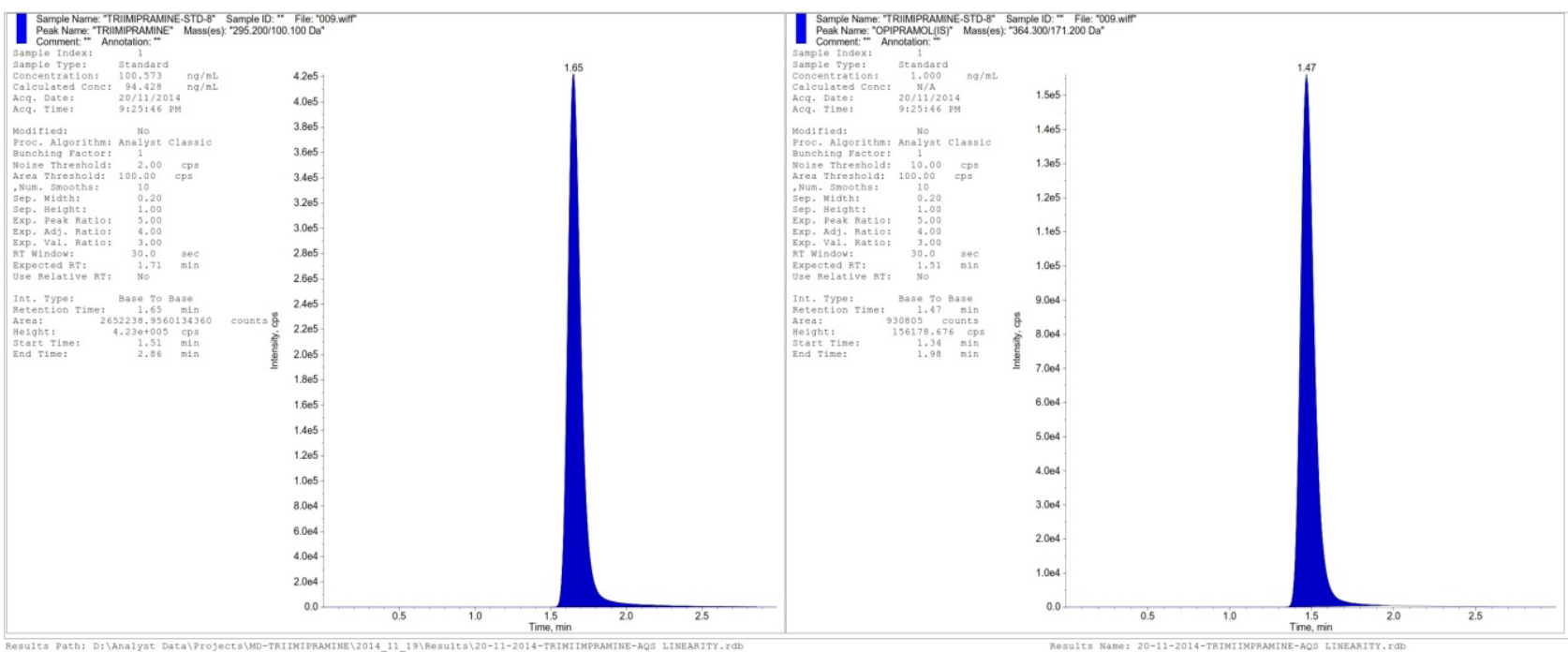

Figure 8: Linearity Standard-8 Chromatogram. 
Citation: Koganti VS, Reddy SS, Thejaswini JC, Bannimath G (2015) Development and Validation of LC-MS/MS Method for Determination of Trimipramine Maleate in Human Plasma. Pharm Anal Acta 6: 413. doi:10.4172/21532435.1000413

Page 6 of 6

\begin{tabular}{|l|l|l|l|l|}
\hline Quality control samples & Concentration added $(\mathbf{n g} / \mathbf{m l})$ & Concentration found(ng/ml) (mean \pm S.D) & Precision (\%) CV \\
\hline LLOQ QC & 0.109 & $0.110 \pm 0.005$ & 4.50 & \\
\hline LQC & 0.284 & $0.270 \pm 0.014$ & 5.19 \\
\hline MQC & 37.809 & $36.013 \pm 0.308$ & 100.91 \\
\hline HQC & 75.619 & $72.361 \pm 0.532$ & 9.85 \\
\hline
\end{tabular}

Table 2: Results for Intra batch Precision and accuracy.

\begin{tabular}{|l|l|l|l|l|}
\hline Quality control samples & Concentration added $(\mathbf{n g} / \mathbf{m l})$ & $\begin{array}{l}\text { Concentration } \\
\text { (mean } \pm \text { S.D) }\end{array}$ & found(ng/ml) & Precision (\%) CV \\
\hline LLOQ QC & 0.109 & $0.110 \pm 0.005$ & 4.50 & 100.91 \\
\hline LQC & 0.284 & $0.270 \pm 0.014$ & 5.19 & 95.18 \\
\hline MQC & 37.809 & $36.013 \pm 0.308$ & 0.85 & 95.24 \\
\hline HQC & 75.619 & $72.361 \pm 0.532$ & 0.73 & 95.7 \\
\hline
\end{tabular}

Table 3: Results for Inter batch Precision and accuracy.

\begin{tabular}{|c|c|c|c|}
\hline \multicolumn{2}{|c|}{ Stability Experiments } & \multirow{2}{*}{ 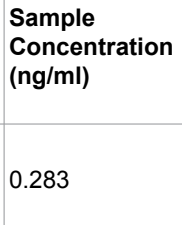 } & \multirow{2}{*}{$\begin{array}{l}\text { Concentration found } \\
\text { (ng/ml) (mean } \pm \text { SD) } \\
0.2731 \pm 0.0078\end{array}$} \\
\hline ze thaw & LQC & & \\
\hline & HQC & 75.518 & $71.7145 \pm 3.0689$ \\
\hline \multirow{2}{*}{$\begin{array}{l}\text { Autosampler } \\
\text { stability }\end{array}$} & LQC & 0.283 & $0.2695 \pm 0.0032$ \\
\hline & HQC & 75.518 & $71.3601 \pm 0.6432$ \\
\hline \multirow{2}{*}{ Bench top } & LQC & 0.283 & $0.2925 \pm 0.0089$ \\
\hline & HQC & 75.518 & $73.9863 \pm 2.6501$ \\
\hline \multirow{2}{*}{ Wet extract } & LQC & 0.283 & $0.3093 \pm 0.0059$ \\
\hline & HQC & 75.518 & $81.6491 \pm 1.0609$ \\
\hline
\end{tabular}

Table 4: Stability studies of the analyte.

extraction technique for the quantification of trimipramine maleate in human plasma using opipramoldihydrochlorideas an internal standard and validated. The desired sensitivity for trimipramine maleate was achieved with an LLOQ of $0.1 \mathrm{ng} / \mathrm{ml}$. The method can be applicable for routine bioavailability/bioequivalence studies and also determination of pharmacokinetics and therapeutic drug monitoring.

\section{Acknowledgment}

Authors are thankful for Clinigene International Ltd, Bengaluru for the facilities provided and also thankful for $R \& L$ fine chemicals for providing the drug.

\section{References}

1. Delgado PL, Miller HL, Salomon RM , Licinio J, Heninger GR, et al. (1993) Monoamines and the mechanism of antidepressant action: effects of catecholamine depletion on mood of patients treated with antidepressants. Psychopharmacol Bull 29: 389-396.

2. Rossi S ( 2013) Australian Medicines Handbook. Adelaide: The Australian Medicines Handbook Unit Trust.

3. Joint Formulary Committee. British National Formulary (BNF). 65 ed. London, UK: Pharmaceutical Press; 2013.

4. Teicher M, Glod C, Cole J (1993) Antidepressant drugs and the emergence of suicidal tendencies. Drug Safety 8: 186-212.

5. Pfuhlmann B, Gerlach M, Burger R, Gonska S, Unterecker S, et al. (2007) Therapeutic drug monitoring of tricyclic antidepressants in everyday clinical practice. Journal of Neural Transmission Supplement 72:287-96.

6. Abdullah A, Gamal AM (2013) Spectrophotometric Determination of Trimipramine in tablet dosage form via charge transfer complex formation,Trop. J. Pharm. Res. 12:1057-1063.

7. Ali RG, Khadijeh S, Payman H, Mir AF (2008) Spectrophotometric determination of Trimipramine in urine after its preconcentration into a microdroplet using homogeneous liquid-liquid extraction. ActaChimSlov. 55:213-218.

8. Rasda B, Paiboon T, Pinit P, Teera K (2013) Development of the method of the Ultra Performance Liquid Chromatography (UPLC) with photo diode array detector for determination of 7 tricyclic antidepressants concentrations in human plasma. Siriraj Medical Journal. 65: 100-104.

9. Product monograph. Trimipramine. Ontario: AA Pharmalnc; 2010. 